\title{
INTEGRATED METHOD FOR COMBUSTION STABILITY PREDICTION
}

\author{
Y. C. Yu, L. O'Hara, R. J. Smith, W. E. Anderson, \\ and C. L. Merkle
}

\author{
Purdue University \\ West Lafayette, Indiana, USA
}

\begin{abstract}
Major obstacles in overcoming combustion instability include the absence of a mechanistic and a priori prediction capability, and the difficulty in studying instability in the laboratory due to the perceived need for testing at the full-scale pressure and geometry to ensure that important processes are maintained. A hierarchal approach toward combustion instability is described that comprises experiment, analysis, and highfidelity computation to develop combustion response submodels that can be used in engineering-level design analysis. The paper provides an illustrative example of how these elements are used to develop a prediction for growth rates in model rocket combustors that generate spontaneous longitudinal combustion instabilities.
\end{abstract}

\section{INTRODUCTION}

Combustion instability arises when the heat addition from an unsteady reacting flowfield energizes the acoustic modes of the combustion chamber and amplifies the associated pressure and velocity oscillations. This is a particularly difficult problem for rocket engines where extreme amounts of energy are released in compact reaction zones in geometries that are mostly closed and have small acoustic losses. The danger of instability is most severe for large rocket engines. They can have multiple modal frequencies in a range that can mechanistically couple with a number of combustion component processes, and the cut-and-try testing methods that have historically been used to solve instability are prohibitively expensive in the modern economy.

Concerns over combustion stability and aversion to risk tend to hinder innovation and cause engineers to rely more on derivative designs. Because the acoustic modes are directly related to the chamber dimensions, there has been a question regarding the value of less expensive subscale test and experiment in

This is an Open Access article distributed under the terms of the Creative Commons Attribution-Noncommercial License 3.0, which permits unrestricted use, distribution, and reproduction in any noncommercial medium, provided the original work is properly cited. 


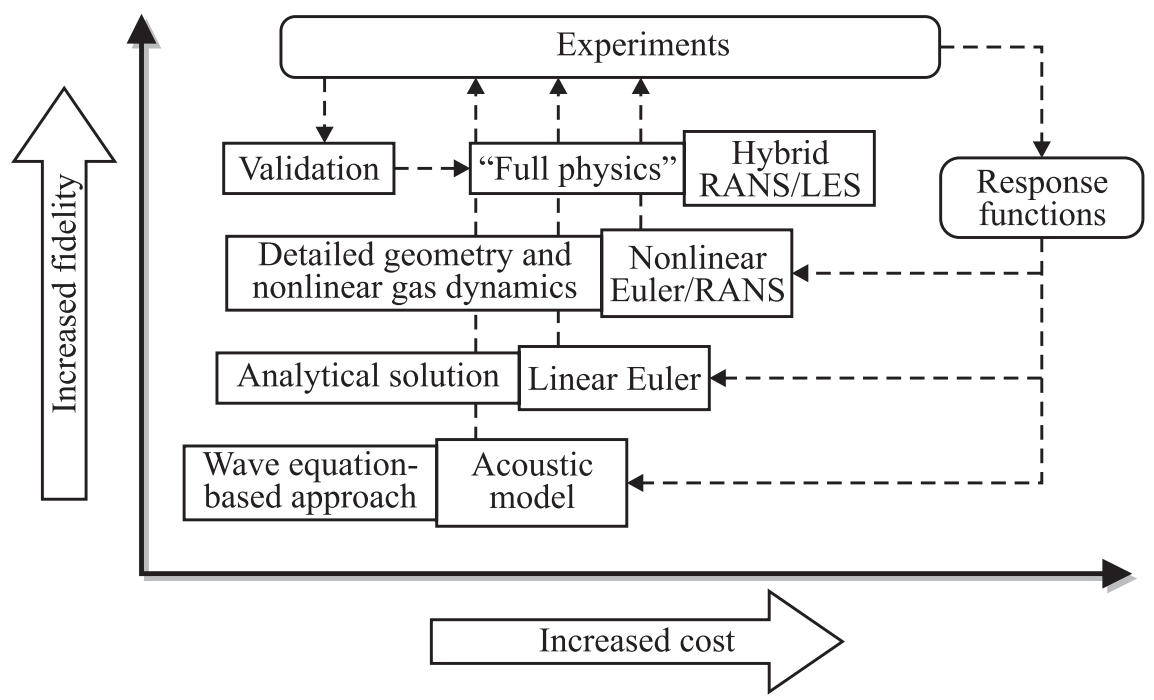

Figure 1 Hierarchal methodology of analysis, computation, and experiment

providing useful design information. Furthermore, the complexity of combustion instability along with computational limits lead to some question whether a priori prediction is even possible. In spite of these valid concerns, progress is critically needed. This paper describes recent results from an ongoing effort to develop a methodology that integrates subscale experimentation, high-fidelity simulations, and engineering analysis to produce substantiated a priori predictions of full-scale rocket-engine combustion instability. A schematic overview of the integrated method is shown in Fig. 1. Understanding the mechanisms of combustion instability is the key to accurate prediction.

It is fairly well-accepted that instability characteristics are, in large part, determined by the injector design and the conditions at which the combustor operates. The present integrated approach starts by choosing a hypothesis regarding the controlling physical mechanisms for the injector of interest and a corresponding experiment that can test the hypothesis. The experimental model should contain the representative injector elements and operate at reasonable conditions. The three elements of experiment, analysis, and simulation are exercised in cycles - the experiment provides physical understanding and validation data for the analyses and simulations, the high-fidelity simulations provide fine details of the physics and allow a quantitative model of the physics, and linear models provide growth rates that are dependent on the output from the high-fidelity model, for comparison to the measured growth rates. To date, this approach has been applied to combustor types based on oxidizer-rich staged-combustion engines $[1,2]$. 
This paper presents a brief description of the integrated approach. An experiment is described that provides data on spontaneous longitudinal instabilities that have been used for validation and understanding. Key results from the experiment, high-fidelity simulation and stationary modeling are summarized in turn. A combustion response $(n-\tau)$ is derived from the simulation, and input to a linear Euler equation analysis for calculation of spatial modes and growth rates which are compared with the results from the detailed simulation and experiment.

\section{RESULTS AND DISCUSSION}

\subsection{Experimental Configuration}

The model combustor examined in this study (Fig. 2) was originally designed to encourage spontaneous longitudinal modes of instability by matching the acoustic modes of the injector tube to those of the combustion chamber. Important features of the design were copied from injectors that are used in oxidizer-rich staged combustion engines. The cross section in Fig. 2 shows the basic elements including an oxidizer tube, a combustion chamber, and a sonic exit, each of which must be modeled to reasonably calculate system resonances. In actual practice, the oxidizer tube is used as a damping device, and full-scale rocket test results have shown the primary importance of whether the inlet is subsonic or sonic and whether the acoustic length of the oxidizer tube corresponds to a $1 / 4$ or $1 / 2$-wavelength of the combustor acoustics, with the subsonic inlet/half-wave oxidizer tube being the most unstable element [3].

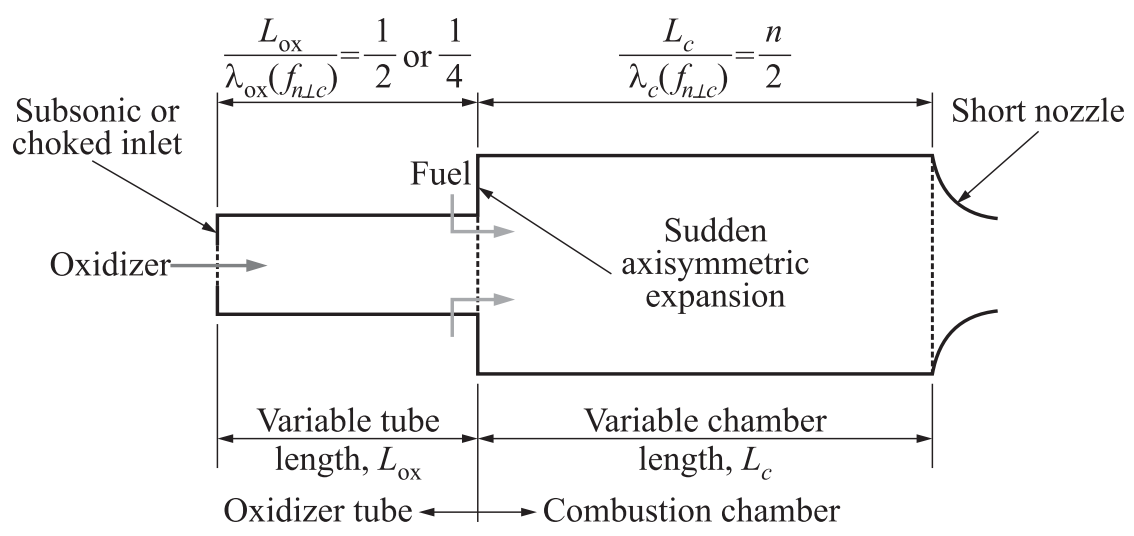

Figure 2 Experimental schematic of spontaneously unstable (longitudinal) combustion chamber 


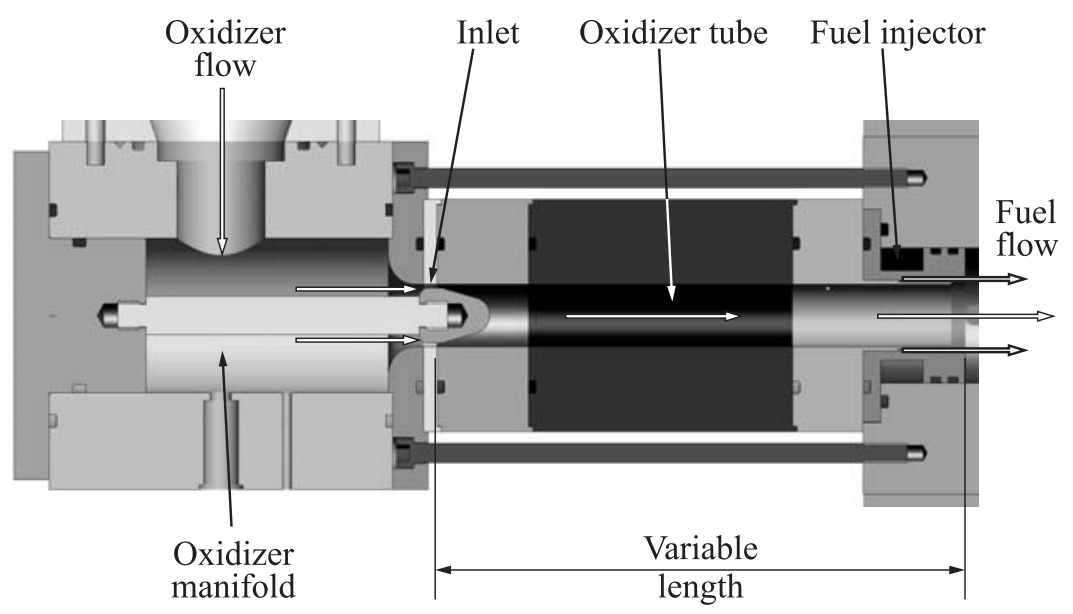

Figure 3 Detailed schematic of choked-inlet injector

Figure 3 shows how the oxidizer and fuel are injected into the combustor in the benchtop experiment. Hot gaseous oxidizer is produced by flowing $90 \%$ (wt.) hydrogen peroxide, $\mathrm{H}_{2} \mathrm{O}_{2}$, through a catalyst bed. The product gas is at a temperature of approximately $1030 \mathrm{~K}$, hot enough to autoignite kerosene-based fuels, and is composed of about $42 \%$ (wt.) oxygen gas, $\mathrm{O}_{2}$, and $58 \%$ (wt.) water vapor, $\mathrm{H}_{2} \mathrm{O}$. The oxidizer is delivered to a manifold and then fed axially into the oxidizer tube. The oxidizer tube length can be adjusted discretely to provide lengths ranging from an acoustic 1/4- to 1/2-wave resonator. Fuel is introduced at the downstream end of the oxidizer tube by orifices that inject the fuel behind a metal collar that is used to initially shield the fuel flow from the high-velocity oxidizer. The propellants which are partially-mixed enter the combustion chamber at an axisymmetric expansion. The chamber length can also be changed discretely to vary resonant frequency. A short converging nozzle is used to maximize reflection of pressure waves in the combustion chamber. The particular experiment discussed here used a swirling flow of JP-8 fuel, a 0.381-meter chamber, a nominal oxidizer-to-fuel mass ratio of 6.2 , and nominal chamber pressure of 2.8 MPa. The nominal oxidizer manifold pressure was $8.3 \mathrm{MPa}$, and the nominal fuel pressure drop was 1.1 MPa.

The oxidizer tube lengths used in the experiment were set based on classical acoustic considerations neglecting the effect of mean flow in the tube, and represented either $1 / 4$ - or $1 / 2$-wave resonators. More recent tests have used a continuously variable tube length [4]. Both sonic (choked) and subsonic inlets have been tested. The choked inlet was an inverted venturi (shown in Fig. 3). In each test, a single high-frequency pressure measurement was made in the oxi- 


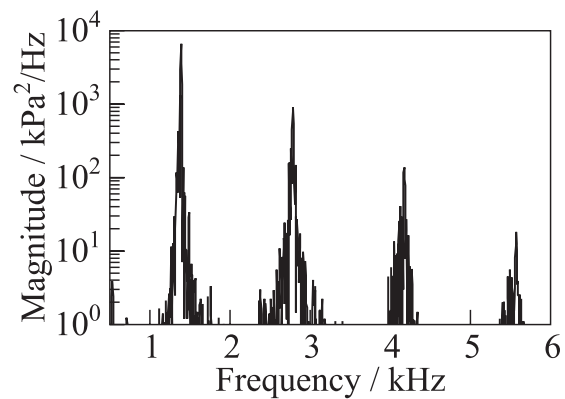

(a)

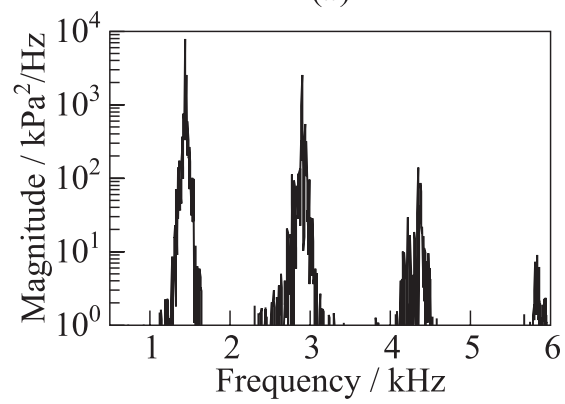

(c)

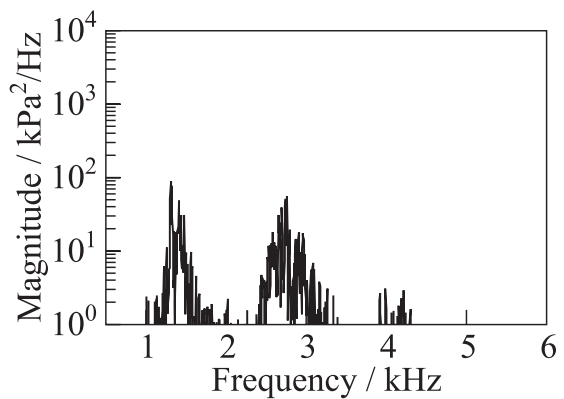

(b)

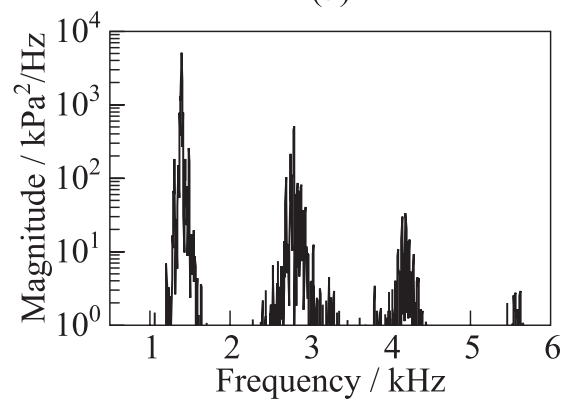

(d)

Figure 4 The PSD computed from $200 \mathrm{~ms}$ of dynamic pressure data measured in 0.381-meter chamber with inlets: (a) 1/2-wave, sonic; (b) 1/4-wave, sonic; (c) 1/2-wave, subsonic; and $(d)$ 1/4-wave, subsonic

dizer manifold and at least two measurements were made in both the combustion chamber and oxidizer tube. Static measurements of mean pressure were made in the oxidizer manifold, oxidizer post, and combustion chamber.

The calculated power spectral density plots are shown in Fig. 4 for the 0.381meter chamber with a combination of $1 / 4$ - and $1 / 2$-wave resonators and sonic (choked) and subsonic inlets. All combinations produced fairly strong instabilities, with the most stable configuration being the $1 / 4$-wave injector tube with a choked inlet, and the most unstable configuration being the $1 / 2$-wave injector tube with a choked inlet. Qualitative trends such as these provide valuable information related to stability mechanisms that can be more thoroughly investigated with high-fidelity computational analyses of each configuration.

Time traces of the high-frequency pressure signal band-pass filtered around $1505 \mathrm{~Hz}$ are shown in Fig. 5. It can be seen that the short period of stable operation that occurs after the autoignition event quickly yields a rapid growth to a high-amplitude limit-cycle pressure oscillation. The figure also shows the initial growth of the instability, from which linear growth rates can be obtained. 


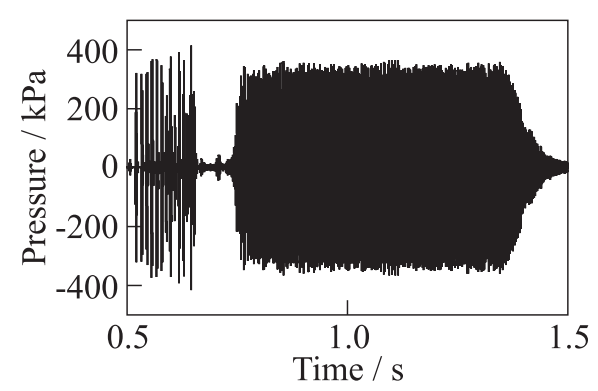

(a)

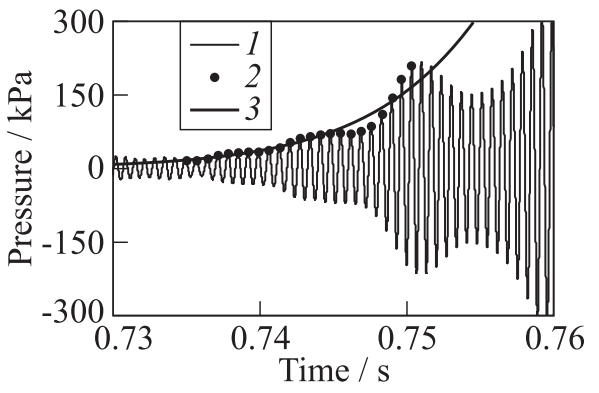

(b)

Figure 5 Band-pass filtered high-frequency pressure signals, $f_{c}=1505 \mathrm{~Hz}(20 \%$ width) measured $0.013 \mathrm{~m}$ downstream of injection plane: (a) time scale begins just prior to ignition and ends just after the fuel flow ceases; and $(b)$ curve-fit growth rate ( $\alpha=145 / \mathrm{s})$ at start of instability $(1$ - filtered data; 2 - curve fit points; and 3 curve fit)

Although the linear growth shown in Fig. $5 b$ may seem to be an excellent source of data for model validation, the repeatable growth rates were not obtained in the experiments. However, the limit-cycle amplitudes are fairly repeatable, and determining linear growth rates using nonlinear models of acoustic mode coupling [5] is the authors' ongoing effort [6].

Figure 6 shows a typical measured waveform at its limit-cycle amplitude and a calculated pressure-time trace using an eight-mode analysis. At this condition, oscillation amplitudes of about $1.0 \mathrm{MPa}$ (36\% of the mean chamber pressure) are measured. The waves are steep-fronted and asymmetric about the aver-

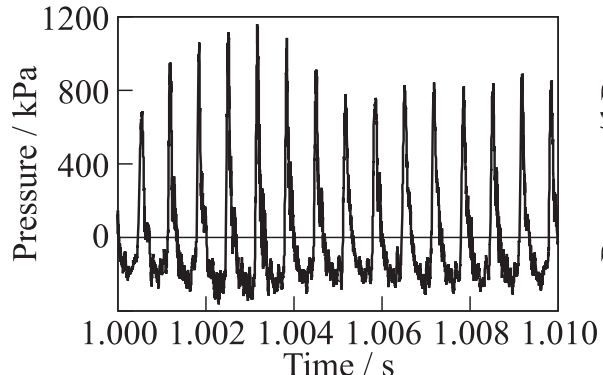

(a)

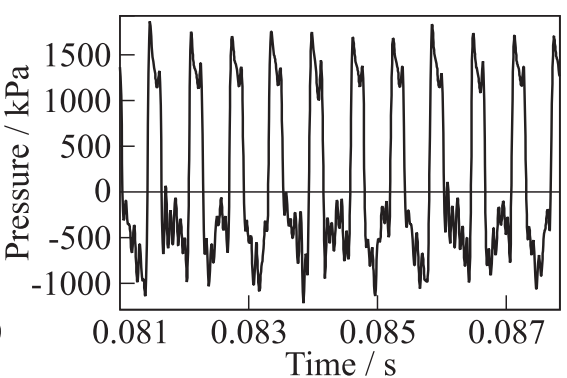

(b)

Figure 6 Limit-cycle wave shapes in 0.381-meter chamber, measured from experiment $(a)$ and calculated by eight-mode nonlinear analysis $(b)$. Instability is at the same frequency 
age pressure amplitude. Comparison between the two is encouraging, but the method needs to be further investigated before reliable linear growth rates can be obtained from the limit-cycle amplitudes.

\subsection{High-Fidelity Computations}

The high-fidelity computations are obtained from numerical solutions of the unsteady Navier-Stokes equations. The computational code used is an in-house implementation with second-order accuracy in both space and time $[7,8]$. The mixing and combustion dynamics are modeled by means of a hybrid RANS/LES (Reynolds-Averaged Navier-Stokes / Large-Eddy Simulation) model [9-11] that computes the large scales directly while the smaller scales are treated by a RANS model. In the boundary layer and near-wall regions, the $k-\omega$ model [12] is used in unmodified form while in regions where large scales can exist, the dissipation term in the turbulence model is diminished according to the ratio between the length scale obtained from the $k-\omega$ model and the local grid size. Both the code and the turbulence model have been verified against numerous fundamental experiments in a wide variety of areas. In particular, combustion instability predictions have been shown to give proper trends concerning changes in combustor back-step heights and upstream boundary conditions [13]. Although the code is formulated for general unstructured grids, simulations are typically run on structured grids with the unstructured format being used to facilitate load-balancing on parallel processors.

The primary advantage of full, unsteady Navier-Stokes instability modeling is that the solutions provide the direct prediction of unsteady heat release and the manner in which it couples with the pressure fluctuations. Thus, whereas in the engineering level analyses the combustion response function must be specified as an input to the modeling, in the hybrid RANS/LES calculations the combustion response function is an output of the simulation. Certainly, this computed response function needs to be validated by comparison with experiment, but it provides a more in-depth model of instability phenomena that can help to guide physical intuition. In addition to predicting the combustion response, the high-fidelity method also provides information on all variables in the flowfield. Those variables that have been measured experimentally can be used to validate the model, while those variables that cannot be measured can be deduced from the simulations and used to augment the understanding provided by the experiment.

In simulations, the computational domain is typically chosen to start from the upstream end of the injector and extend to downstream of the choked throat. A representative computational domain is shown in Fig. 7. In this figure, the oxidizer is injected radially on the left end, then goes through a choked, slotted inlet so that pressure fluctuations from the chamber cannot couple with the 


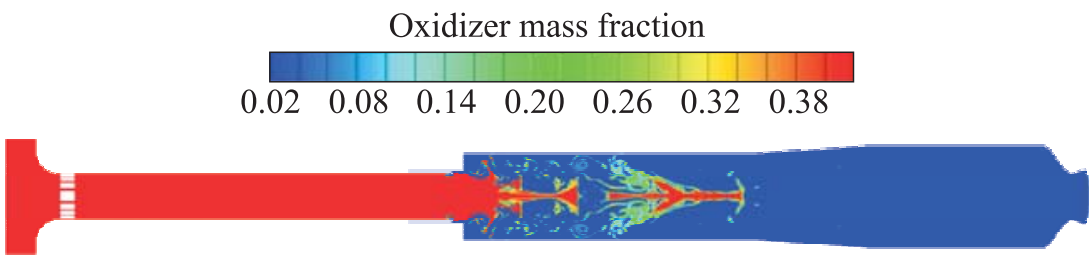

(a)

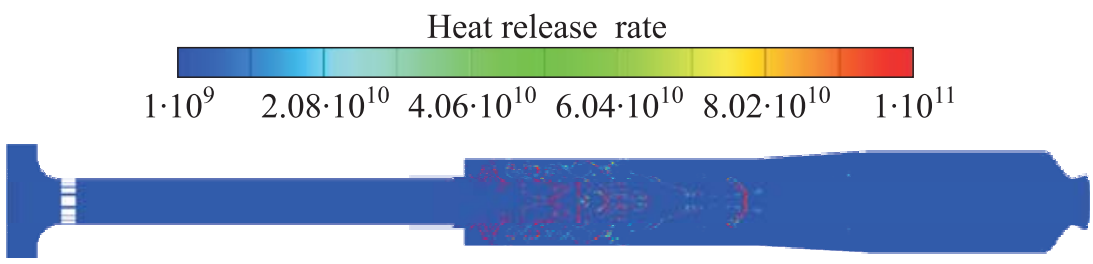

(b)

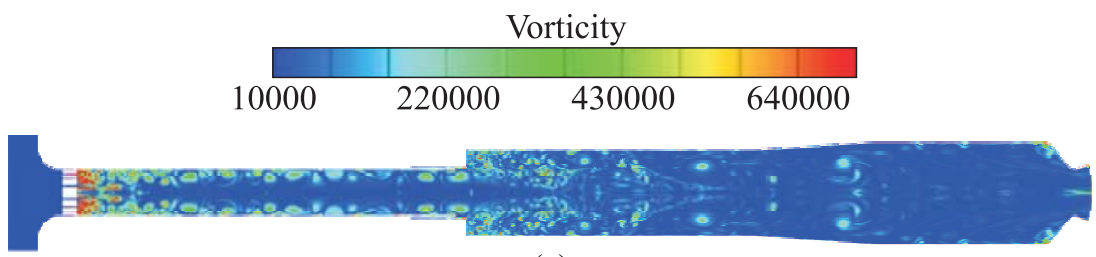

(c)

Figure 7 Representative instantaneous contours of oxidizer mass fraction $(a)$, heat release rate $(b)$, and vorticity $(c)$ approximately $100 \mathrm{~ms}$ after start of simulation [14]. (Refer Yu et al., p. 304.)

manifold. The oxidizer then flows downstream and is eventually joined by injected fuel just upstream of the back-step at the entrance to the main combustion chamber.

The instantaneous snapshots of the flowfield shown in Fig. 7 are taken approximately $100 \mathrm{~ms}$ after flow initiation when it has been verified that the effects of initial conditions and startup transient have been washed out of the computational domain and stationary unsteady conditions have been set up. The contour plot of Fig. $7 a$ shows the oxidizer concentration, whereas Fig. $7 b$ shows the instantaneous heat release, and Fig. $7 c$ shows the vorticity at one instant of time. Note that the oxidizer begins to roll up as it enters the main combustor, giving rise to rapid mixing and combustion. The combustion front itself is seen to be distributed across the front half of the combustor in a very distributed and transient manner. The instantaneous vorticity shows that it is produced at the inlet plug and the back-step where the flow enters the combustor and then propagates downstream to the axisymmetric expansion where its scale is changed and it interacts with the incoming fuel and, subsequently, the wall. 


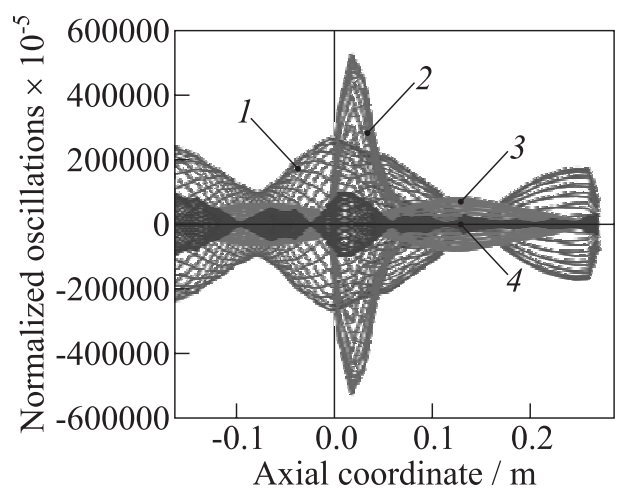

Figure 8 Composite plot of pressure (1), heat release (2), axial velocity (3), and vorticity (4) fluctuations throughout the oxidizer post and combustion chamber at a series of times over one period [14]

Figure 8 shows a composite plot of the acoustic pressure, vorticity fluctuation, instantaneous heat release, and axial velocity in the injector post/combustor combination (note that vorticity and axial velocity fluctuations have been scaled to be comparable with pressure and heat release fluctuations). These results have been obtained from the hybrid RANS/LES calculations, filtered at the first longitudinal (1L) mode, down-sampled, and cycle-averaged to show conditions at 28 equally spaced time intervals over one acoustic cycle. Before plotting, each variable was averaged across the radius to give an average value at each spatial location at that particular time in the cycle. The result is a series of "mode shape" plots which show how pressure, axial velocity, vorticity, and heat release vary in time and space. The $1 \mathrm{~L}$ mode is easily recognized from the pressure traces that show a node near the center of the combustor and antinodes at the step and the combustor exit. Note that the $1 \mathrm{~L}$ combustor mode is coupled with a similar mode in the oxidizer post. There is not a sharp pressure node in the center of the oxidizer post because of the higher Mach number in this narrower channel. The fluctuations of the axial velocity are nearly opposite to those of the pressure, with velocity nodes at pressure antinodes and velocity antinodes at pressure nodes. These mode shapes can be directly compared to those from acoustics or Euler-equation models.

The heat release fluctuations are concentrated over the first one-fourth of the combustor. The heat release and vorticity amplitudes are larger for the smaller step-height combustor, with nodes and antinodes analogous to those seen in the vorticity fluctuations [13]. In particular, the peak vorticity fluctuation occurs near the same location as the peak heat release, an indication that the heat release is driven by vorticity generation at the step. Analyses of individual time intervals in this plot show that the pressure and heat release fluctuations are 
largely in phase, suggesting that the heat release is driving the disturbances [14]. It is emphasized that in both the numerical simulations and in the companion experiments, the instability was generated naturally from the combustion process and the chamber acoustics. External forcing was not employed.

Before using the results shown in Fig. 8 to develop a model of combustion response, the simulation tool must be validated using experimental data. In contrast to steady-flow simulations where detailed, multidimensional experimental measurements can be readily obtained for direct quantitative validation of steady computations, transient experiments like the present one do not have the luxury of obtaining sufficient temporal and spatial resolution to provide direct validation of a time-accurate simulation. Instead, the simulation must be validated by comparing trends and observed differences in behavior as measured in different chambers or at different operating conditions. Further, it is unrealistic to expect a simulation to provide quantitative accuracy in terms of disturbance amplitudes and/or growth rates. Accordingly, the validation procedure for the present integrated approach was to compare experimentally observed trends with similar trends predicted by the simulations. As noted above, to date the qualitative effects of both chamber diameter and upstream inlet configuration on disturbance amplitude have been successfully predicted. These qualitative trends were accomplished by comparing such quantities as the rudimentary resonant frequencies, spatial mode shapes, and phase angles between high-frequency pressure measurements made at different chamber locations. In addition to experimental validation, the precise growth rates obtained from the analytical model can, and were, used as verification of the numerical approach.

An important aspect of the high-fidelity simulations is that appropriate postprocessing procedures must be developed to extract maximum physical understanding from the myriad of numbers that are provided by a high-resolution, unsteady computation. Representative postprocessing steps in use now include Power Spectral Density (PSD) analyses of stationary time series to deduce dominant frequencies in the simulations, band-pass filtering of the solutions at specific peaks in the PSD's to visualize the mode shapes, companion phase analysis of lag/lead between pressure, heat release, vorticity, etc. as noted earlier, and computation of Rayleigh indices to document the degree of combustion driving. Developing techniques such as these to analyze the simulation results remains a challenging problem. In keeping with the authors' overall goal, an important step is to develop improved methods for extracting combustion response functions from the simulations. Representative response functions obtained by combining the hybrid RANS/LES calculations with the analytical analyses are discussed below.

A test of the hypothesis that vortex shedding is a controlling process in the instability was done by studying the effect of combustor step height at the sudden expansion (see Fig. 2). It was observed experimentally that the large step height was more stable. A plot of the time history of the simulated Rayleigh index 


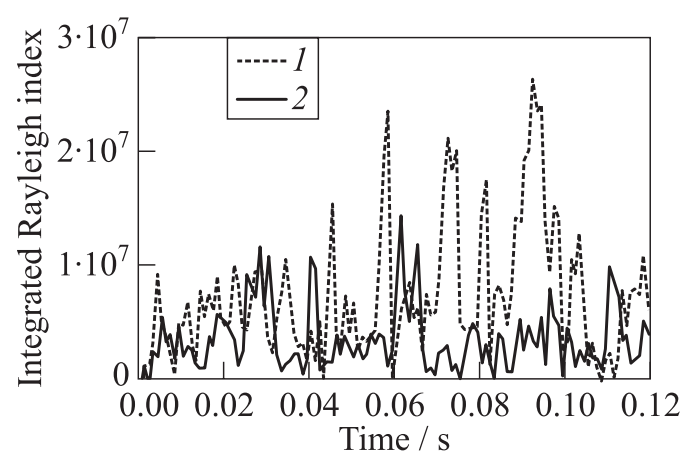

Figure 9 Axially integrated Rayleigh index calculated at 1.0-millisecond intervals for two step heights [14]: 1 - smaller step; and 2 - larger step

for chambers with two different back-step heights is shown in Fig. 9. This plot covers the entire range from the initial quiescent conditions in the chamber to the stationary, unsteady condition. The time required to reach stationary behavior in this case is approximately $50 \mathrm{~ms}$. These Rayleigh indices are integrated over the entire combustor volume at each instant of time. Note that, in general, the smaller chamber shows a larger Rayleigh index than does the larger one, although there are regions where it is smaller than the Rayleigh index for the larger chamber. In addition, it is seen that the magnitude of the Rayleigh index varies widely with time and that there is no readily discernable pattern.

As a final result from the high-fidelity model, Fig. 10 shows the PSD of the pressure for the same two chambers. The spectrum shows a clear peak at approximately $1750 \mathrm{~Hz}$ (the 1L mode). In addition, the higher harmonics are also shown. Again, the PSD results suggest stronger pressure fluctuations for the chamber with the smaller back step. This trend is in keeping with Rayleigh index predictions that showed stronger driving in the smaller chamber. Additionally, it agrees with the experimental measurements that indicated the smaller chamber is more unstable than the larger chamber.

\subsection{Analysis}

It is of great practical importance to apply the physical insight provided by the high-fidelity simulation results across a range of operational and geometric conditions. Simulations require a great amount of run time; so, design analysis should be conducted using lower-order models. A preliminary investigation into modeling the results of high-fidelity simulations to provide input to stability analysis is described below. 


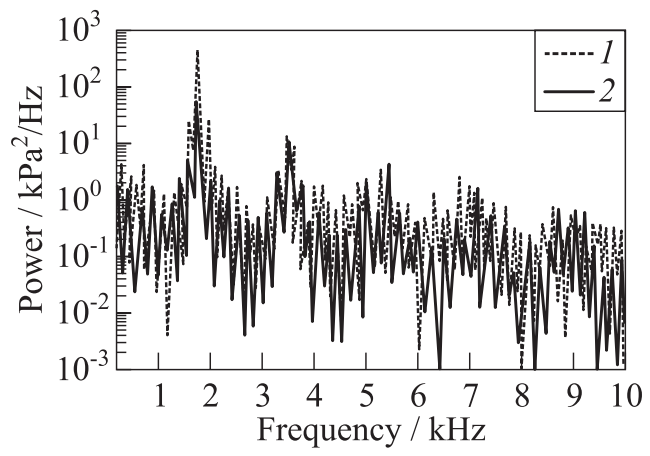

Figure 10 The PSD analysis of hybrid RANS/LES results over an interval of 70 to $120 \mathrm{~ms}$ [14]: 1 - smaller step; and 2 - larger step

Acoustic models should be limited to low-Mach-number applications that have boundaries corresponding well to acoustic approximations. The linearized Euler equations (LEE) model including mean flow effects and entropy waves is formulated for multiple-domains to account for mean-flow property changes, and is generalized for physically-realistic boundary conditions to allow direct comparisons with higher-order computational fluid dynamics (CFD) models. The reduced-order results are cost-efficient and can be easier to understand, and provide exact analytical solutions that can be used for verifying higher-order models for simple geometries. The LEE model has been systematically exercised to investigate various flow effects and boundary conditions [4].

The output from the high-fidelity analysis can be used to derive combustion response models for input to the lower-order models, which can then be used to conduct a more expansive study. In addition to providing an approximation of how the combusting flowfield interacts with the unsteady flowfield, ideally the combustion response model provides a good physical representation of the coupling process. In the absence of this mechanistic model, a simple pressurecoupled time-lag model is used:

$$
q^{\prime}(x, t)=n(x) \frac{\bar{q}}{\bar{p}} p^{\prime}(x, t-\tau(x))=N(x) p^{\prime}(x, t-\tau(x)) .
$$

The RANS/LES calculation results are used to determine the spatiallyvarying phase difference between unsteady heat release and pressure fluctuations, $\tau(x)$, and interactive index, $N(x)$. The calculated parameters are averaged between $40-70 \mathrm{~ms}$, during the linear growth regime of combustion instability. Calculated time lag and interactive index are plotted in Fig. 11.

The calculated values of $N$ and $\tau$ are used with geometric and mean flow input in the LEE model. The computation is divided into ten domains shown in 


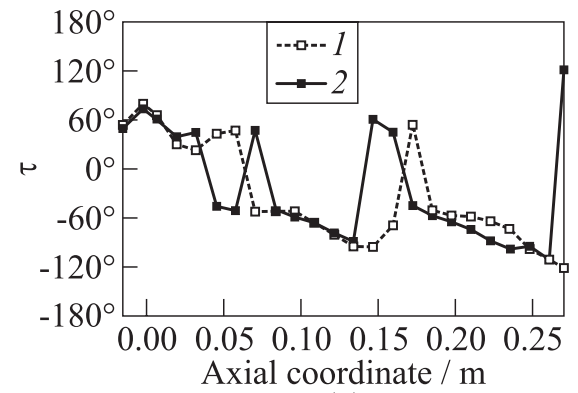

(a)

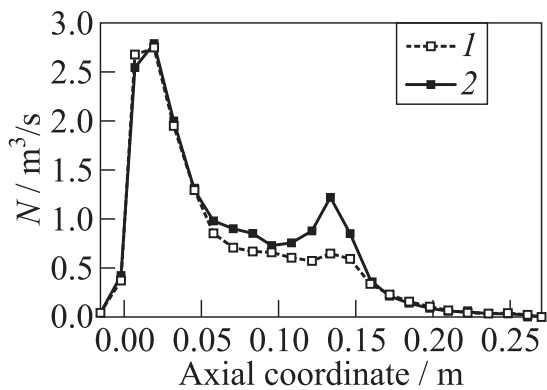

(b)

Figure 11 Spatially-varying time-lag $(a)$ and interactive index $(b)$ determined from RANS/LES calculations, averaged over the interval 40-70 ms: 1 - smaller step height; and 2 - large step height

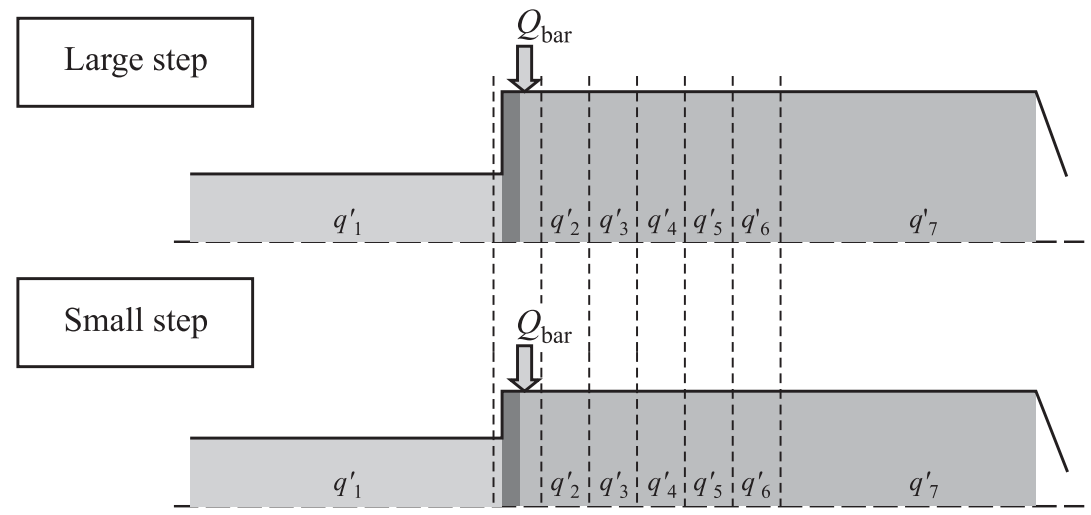

Figure 12 Schematic of LEE-model computational domain (10 domains, 3 for mean flow, 7 for $q_{\text {LES }}^{\prime}$ ). (Refer color plate, p. XXX.)

Fig. 12. Three domains were used to account for the mean-flow property change due to geometry and mean heat addition, indicated by different color. Mean heat addition is applied at 0.5 -inch downstream of the injector face $\left(Q_{\text {bar }}\right)$, according to the RANS/LES results. Five domains are used to approximate the diverging sections in the combustor section.

Seven domains were used to apply the unsteady heat release, represented by the dashed lines. From the RANS/LES results, unsteady heat release is concentrated over the first half of the combustor section, with unsteady heat release added at $-0.00635\left(q_{1}^{\prime}\right), 0.01905\left(q_{2}^{\prime}\right), 0.04445\left(q_{3}^{\prime}\right), 0.06985\left(q_{4}^{\prime}\right), 0.09525$ $\left(q_{5}^{\prime}\right), 0.12065\left(q_{6}^{\prime}\right)$, and $0.14605 \mathrm{~m}\left(q_{7}^{\prime}\right)$, in accordance with the spatially-varying time-lag acquired from the high-fidelity model. 
The LEE model calculates resonant frequencies, linear growth rates, and spatial mode shapes. Without the addition of unsteady heat release, the LEE model cannot identify the most unstable mode. With the incorporation of an unsteady heat release model using spatially-varying $N(x)$ and $\tau(x)$, the LEE model shows signs of identifying the most unstable mode.

For the LEE model to produce growth rates comparable to the experiment, the spatially-varying $N(x)$ derived from the simulations are multiplied by a factor of 1.8. The LEE analysis confirms the observed trend of relative stability between the two experimental cases in terms of the calculated linear growth rate.

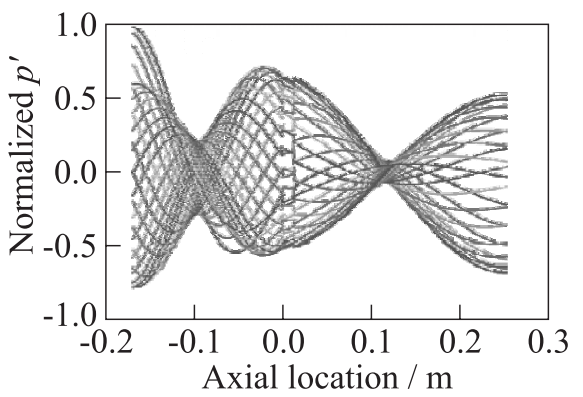

(a)

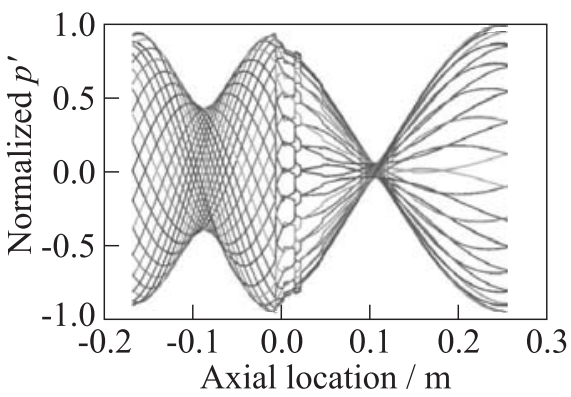

(b)

Figure 13 Spatial mode shapes for the smaller step, $p^{\prime}(x, t)$, calculated from LEE model: (a) without unsteady heat-release response function: $\omega=1947 \mathrm{~Hz}$ and $\alpha$ $\left.=-962 \mathrm{~s}^{-1}\right)$; and $(b)$ with spatially-varying unsteady heat-release model $(\omega=1802 \mathrm{~Hz}$ and $\alpha=145 \mathrm{~s}^{-1}$ )

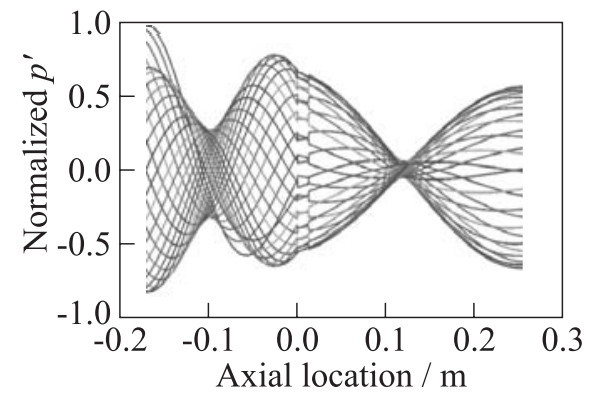

(a)

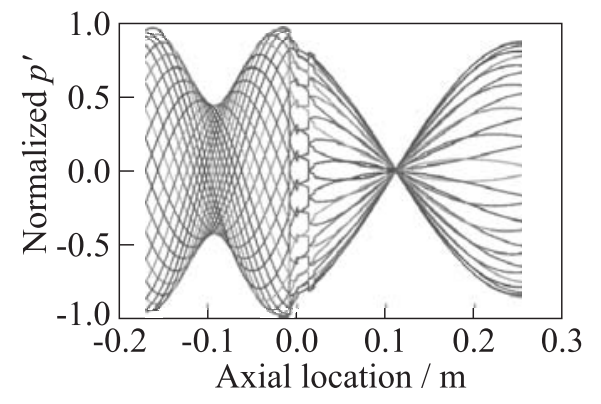

(b)

Figure 14 Spatial mode shapes for the larger step, $p^{\prime}(x, t)$, calculated from LEE model: (a) without unsteady heat-release response function: $\omega=2006 \mathrm{~Hz}$ and $\alpha$ $=-710 \mathrm{~s}^{-1}$ ); and (b) with spatially-varying unsteady heat-release model: $\omega=1889 \mathrm{~Hz}$ and $\alpha=84 \mathrm{~s}^{-1}$ ) 
Including the unsteady heat response function also results in a shift in resonant frequency (within 6\%).

It is also interesting to note that the unsteady heat-release response function helps the agreement between the calculated and measured spatial mode shape. The LEE spatial mode shapes for both small and large step-height configurations are shown in Figs. 13 and 14, respectively. Better agreement between the calculated spatial mode shapes is achieved when unsteady heat release is added to the linear model. The RANS/LES spatial mode shapes for the small-step configuration (see Fig. 8) show that the magnitude of the pressure antinode at the inlet is lower than that at the injector face. In the LEE calculation with no heat release model, the magnitude of pressure antinode at the inlet is higher than at the injector face. However, when the spatially-varying unsteady heat release is added, the relative amplitudes are about the same and agree well with the high-fidelity simulation. The addition of an unsteady heat release response function led to a sharper pressure node in the combustor section, which agrees better with the RANS/LES results.

\section{CONTINUOUS VARIATION OF INJECTOR TUBE LENGTH}

The experimental results showed the importance of injector tube length. More recent experiments included the use of a traversing choked inlet to continuously vary the injector tube length during the test [4]. One of the primary objectives of the traversing experiments was to examine the combustion instability characteristics under continuously variable resonance conditions (CVRC). These tests were conducted with gaseous methane as the fuel to simplify the combustion modeling by eliminating the complicating factor of two-phase flow.

The spectrogram depicted in Fig. 15 shows how the instability develops while the length of the injector tube is shortened from 0.1905 to $0.0953 \mathrm{~m}$. The combustor length was kept fixed at $0.381 \mathrm{~m}$ for all the tests with gaseous methane. To initiate autoignition with gaseous oxidizer, a small quantity (25 ml) of kerosene was injected prior to gaseous methane. The liquid fuel was calculated to be consumed in less than $80 \mathrm{~ms}$.

After the transient startup behavior, the choked inlet was held stationary for $1.5 \mathrm{~s}$ to ensure that a limit cycle has been reached. Both forward- and backward-traversing tests have identified the same transitional points between stability regions. The stability behavior and limit-cycle amplitudes were apparently independent of traverse direction. The strongest instabilities occurred at intermediate lengths, whereas low to moderate instabilities were observed at the longer and shorter lengths. Repeatability tests confirmed the limit-cycle amplitudes and transition points. 


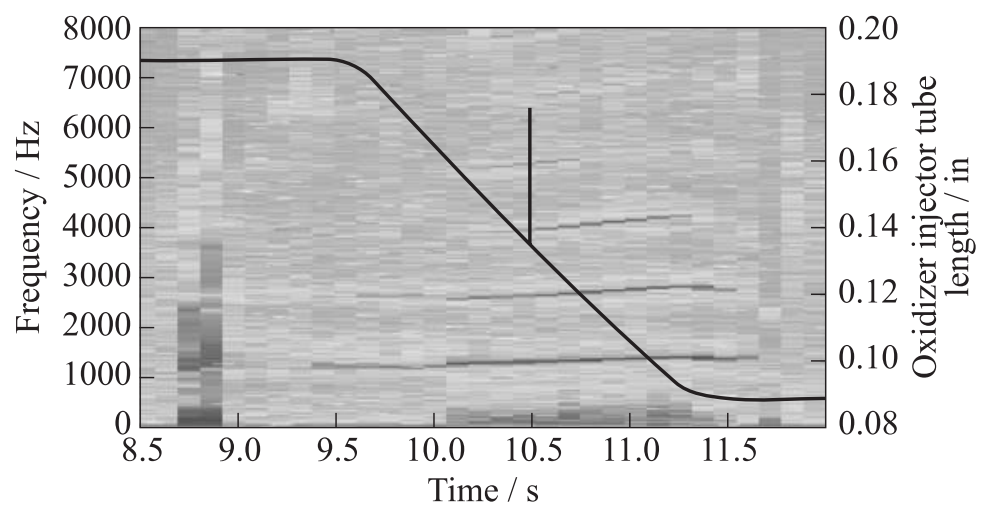

Figure 15 Spectrogram of high-frequency pressure measured $38.1 \mathrm{~mm}$ (1.5 in) downstream of combustor inlet as length of oxidizer tube varies from 0.1905 to $0.0953 \mathrm{~m}$. Deep red indicates high amplitude

To investigate whether nonlinearity had a gross effect on stability, tests were conducted with oxidizer tube lengths fixed at 0.1905, 0.1651, 0.1397, 0.1016, and $0.0953 \mathrm{~m}$. These tests verified that the limit-cycle amplitudes were comparable to the quasi-stationary values under traversing conditions. They could also provide linear growth rates, which are crucial validation parameters for the LEE analysis under different resonance conditions.

The linear growth rates were obtained using an exponential curve-fit to the band-pass filtered pressure trace. The experimental fundamental frequency, linear growth rates, $R^{2}$ values for exponential curve-fit functions, and averaged limit-cycle amplitudes are summarized in Table 1. The limit-cycle amplitudes are fairly repeatable and the linear growth rates are comparable, except for the

Table 1 Summary of experimental results at various fixed injector-tube lengths

\begin{tabular}{ccccc}
\hline $\begin{array}{c}\text { Oxidizer injector } \\
\text { tube length, m }\end{array}$ & $\begin{array}{c}\text { Fundamental } \\
\text { frequency, Hz }\end{array}$ & $\begin{array}{c}\text { Linear growth } \\
\text { rates, 1/s }\end{array}$ & $\begin{array}{c}R^{2} \text { values for } \\
\text { curve-fit }\end{array}$ & $\begin{array}{c}\text { Limit-cycle } \\
\text { amplitude, kPa }\end{array}$ \\
\hline 0.1905 & 1280 & - & - & 13.79 \\
0.1651 & 1260 & 164.8 & 0.9662 & 241.32 \\
0.1651 & 1290 & 148.2 & 0.9136 & 227.54 \\
0.1397 & 1300 & 270.0 & 0.9649 & 220.64 \\
0.1397 & 1330 & 246.6 & 0.9644 & 213.74 \\
0.1016 & 1370 & 318.3 & 0.9634 & 131.01 \\
0.1016 & 1410 & 178.7 & 0.9411 & 144.79 \\
0.0953 & 1360 & 36.6 & 0.6260 & 27.58 \\
\hline
\end{tabular}




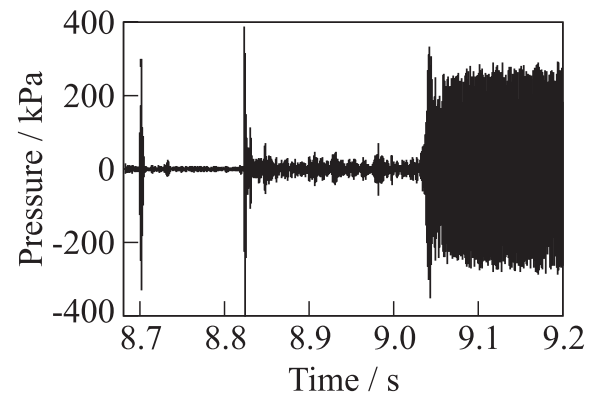

(a)

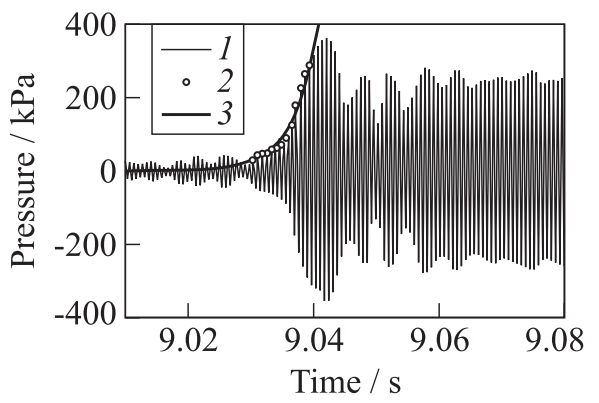

(b)

Figure 16 Band-pass filtered high-frequency pressure signals, $f_{c}=1330 \mathrm{~Hz}$ (20\% width) measured $0.0381 \mathrm{~m}$ downstream of injection plane: $(a)$ time scale begins just prior to ignition and ends after limit cycle has been reached; and $(b)$ curve-fit growth rate $\left(\alpha=247 \mathrm{~s}^{-1}\right)$ at start of instability $(1-$ filtered data; 2 - curve fit points; and 3 - curve fit)

linear growth rate at an injector tube length of $0.1016 \mathrm{~m}$. No linear growth rates were obtained at an oxidizer tube length of $0.1905 \mathrm{~m}$, where the system was marginally stable. These results confirmed the limit-cycle amplitude and transition point trends observed in the translating experiments, and provided important validation information.

The experimental pressure trace, band pass filtered around the fundamental frequency $\left(f_{c}=1330 \mathrm{~Hz}, 20 \%\right.$ width), is presented in Fig. 16. The first pressure spike corresponds to the ignition initiation, and the second pressure spike (130 ms

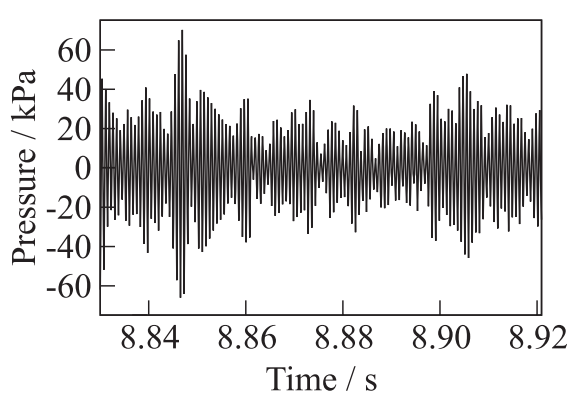

(a)

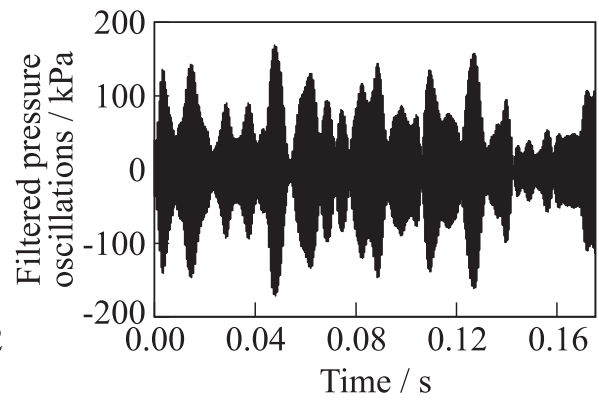

(b)

Figure 17 Comparisons between band-pass filtered pressure signals: (a) measured pressure oscillations with time scale beginning right after ignition transient for $91 \mathrm{~ms}$; and $(b)$ computed band-pass filtered pressure trace from high-fidelity simulations 
later) corresponds to the introduction of gaseous methane. Figure $16 a$ shows a period of relatively stable behavior for $200 \mathrm{~ms}$ after the initial ignition transient, before a large-growth event occurs. Figure $16 b$ provides the initial growth of the pressure signal at the fundamental mode and the exponential curve-fit data which is used to obtain the linear growth rates.

An interesting experimental observation is that linear growth occurs after more than 200 cycles with growth and decay. A zoom view of the band-pass filtered high-frequency pressure signals at the time scale in accordance to the high-fidelity simulation is shown in Fig. $17 a$. Figure $17 b$ shows the band-pass filtered computed pressure at an oxidizer injector tube length of $0.1397 \mathrm{~m}$. The observed behavior prior to linear growth is also seen in the high-fidelity simulations. However, the computed pressure from simulation oscillates at magnitudes a factor of three higher than the measured pressure. No large growth event has been observed yet from the high-fidelity simulations. Detailed comparisons between the experiments and simulations are ongoing.

\section{SUMMARY AND CONCLUDING REMARKS}

The integrated use of experiments, analysis, and high-fidelity simulations in the prediction and description of combustion instability was described. Using the same variables to test the simulation and the analysis that are used in the experiments provides an opportunity for validation and to gain experience using results from high-fidelity simulations to produce combustion response models for use in linear analysis. The model unstable combustor comprised a variable geometry oxidizer tube and sudden expansion combustor. Spontaneous longitudinal instabilities were generated that were dependent on the length of the injector tube and the combustion chamber diameter. Detailed results from a hybrid RANS/LES were used to derive an $n-\tau$ unsteady heat release model that was input to a linear model to calculate growth rates.

Growth rates calculated by the model were compared with those measured in the experiment. An experiment that tested the effect of the continuous variation of the injector tube geometry on the limit cycle behavior of the instability was described. Another set of experiments was conducted at fixed length and showed that the injector tube geometries producing high measured rates of growth in the fixed-length tests also produced high limit-cycle amplitudes in the traversing experiments.

In addition to the prescribed resonance conditions from the experimental geometry, combustion mechanism is a key issue. For liquid kerosene-based fuel, the 1/2-wave choked injector demonstrates high levels of instability; however, this geometric combination corresponds to the most stable configuration for gaseous methane. 
Important areas for future study include development of mechanistic combustion response models and methods for deriving data from the high-fidelity simulations.

\section{ACKNOWLEDGMENTS}

This work was sponsored by AFOSR Contract FA9550-08-C-0033, with Dr. Mitat Birkan as the project manager and Dr. Douglas Talley as technical monitor, and NASA Constellation University Institutes Project under grant NCC3-989 with Ms. Claudia Meyer as the project manager. Significant contributions were also made by Dr. James Sisco of Aurora Flight Sciences, Dr. Enrique Portillo of Siemens Energy, Dr. Sankaran Venkateswaran of NASA Ames Research Center, and Dr. Guoping Xia of Purdue University.

\section{REFERENCES}

1. Miller, K., J. C. Sisco, N. Nugent, and W.E. Anderson. 2007. Combustion instability with a single-element swirl injector. J. Propul. Power 23(5):1102-12.

2. Sisco, J. C., Y.C. Yu, V. Sankaran, and W. E. Anderson. 2011. Examination of mode shapes in an unstable model combustor. J. Sound Vibr. 300(1):61-74.

3. Dranovsky, M. L. 2007. Combustion instabilities in liquid rocket engines: Testing and development practices in Russia. Eds. V. Yang, F. E. C. Culick, and D. G. Talley. Progress in aeronautics and astronautics. Washington: AIAA. 221.

4. Yu, Y., L. O'Hara, J. Sisco, and W. Anderson. 2009. Experimental study of highfrequency combustion instability in a continuously variable resonance combustor (CVRC). 47th AIAA Aerospace Sciences Meetings Proceedings. Orlando, FL.

5. Paparizos, L. G., and F. E. C. Culick. 1989. The two-mode approximation to nonlinear acoustics in combustion chambers. I. Exact solution for second order acoustics. Combust. Sci. Technol. 65:39-65.

6. Sisco, J., J. Portillo, Y. Yu, and W. Anderson. 2007. Non-linear characteristics of longitudinal instabilities in a model rocket combustor. AIAA Paper No. 2007-5570.

7. Venkateswaran, S., and C. L. Merkle. 1995. Dual-time stepping and preconditioning for unsteady computations. AIAA Paper No. 1995-0078.

8. Li, D., S. Venkateswaran, K. Fakhari, and C. L. Merkle. 2001. Convergence assessment of general fluid equations on unstructured hybrid grids. AIAA Paper No. 2001-2557.

9. Travin, A., M. Shur, M. Strelets, and P. R. Spalart. 2000. Physical and numerical upgrades in the detached-eddy simulation of complex turbulent flows. In: 412 EUROMECH Colloquium on LES of Complex Transitional and Turbulence Flows: Book of abstracts. Munich. 
10. Menter, F. R. and Y. Egorov. 2005. A scale-adaptive simulation model using twoequation models. AIAA Paper No. 2005-1095.

11. Basu, D., A. Hamed, and K. Das. 2005. DES, hybrid RANS/LES and PANS models for unsteady separated turbulent flow simulations. FEDSM'05 Proceedings. FEDSM2005-77421

12. Wilcox, D. C. 1998. Turbulence modeling for CFD. 2nd ed. La Canada, CA: DCW Industries.

13. Smith, R., G. Xia, W.E. Anderson, and C. L. Merkle. 2010. Computational simulations of the effect of backstep height on nonpremixed combustion instability. AIAA J. 48(9):1857-68.

14. Smith, R., G. Xia, W. E. Anderson, and C. L. Merkle. 2010. Exploration of combustion instability mechanisms in computational simulations of a longitudinal model rocket. AIAA Aerospace Sciences Meeting. 